\title{
The IDI Classification of Crohn's Disease
}

\author{
Gilles R G Monif* \\ Infectious Diseases Incorporated, US
}

Submission: January 26, 2021; Published: February 10, 2021

*Corresponding author: Gilles R G Monif, Infectious Diseases Incorporated, Bellevue, Nebraska, US

Keywords: Crohn's disease; Pathogenesis; Gastrointestinal bacterial flora; Mycobacterium avium subspecies paratuberculosis; Mucosa; Small intestines; Lamina propria; Biologics; Steroids; Anastomosis; Anaerobic bacterial; Homogenous disease; Enterobacteriaceae

\section{The IDI Classification of Crohn's Disease}

The IDI (Infectious Diseases Incorporated) classification of Crohn's disease is a distillate of nearly 20 years of studying of Mycobacterium avium subspecies paratuberculosis and Crohn's diseases. Unraveling the events that combine to produce Crohn's disease has allowed for the construction of a disease classification based on the disease's pathogenesis rather than disease severity. Over-emphasis of disease's immune-mediated character and insufficient focus on the invasive gastrointestinal bacterial flora has created therapeutic ambiguity.

\section{Rational for the Classification}

The initial destructive phase within Crohn's disease is the consequence of an immune-mediated interaction between Mycobacterium avium subspecies paratuberculosis (MAP)'s directed,Th1 pro-inflammatory cytokines and MAP antigen concentrated in the ileocecal region of the small intestines [1,2]. The net result of this inter-reaction is focal destruction of the lining mucosa. The loss of mucosal integrity allows for bacterial penetration into the underlying lamina propria [3,4]. With $80 \%$ of host immunity being dedicated to the gastrointestinal tract, the underlying lamia propria can withstand a surprising degree of microbial invasion. When administered alone, biologics or steroids can often achieve temporary clinical remission. This ability to induce temporary remissions without antibiotic coverage has argued for mucosal healing as being central to limiting the tissue destructive consequences of the secondary polymicrobial bacterial invasion. Bowel-to-bowel anastomosis, bowel penetration and perineal fistula are the direct consequences of sustained anaerobic bacterial replication within the musculature of the small bowel.

\section{Need for Classification}

Attaining statistical proof of concept in Crohn's disease has required the lumping together of individuals with diverging disease status. The assumption that the label of Crohn's disease identified a homogenous disease entity has resulted in Class IA, Class 1B, Class 2A and Class 2B being intermixed with an occasional Class 3 individual. While suggesting an overall trend, the amalgamation of disease status has tended to mask the underlying specifics. The objective of any classification system is to render order.

\section{Class 1 Crohn's Disease}

Class 1 Naïve Crohn's Disease: Inflammatory loss of mucosal integrity with microbial containment by local immunity. Rapid reestablishment of mucosal integrity (mucosal healing) resulting in temporary remissions can be readily attained with several therapeutic regimens (biologics, steroids, dietary manipulation, etc.).

Class 1A Naïve Crohn's Disease: Under contained polymicrobial infection. Some overt markers of inflammation (CRP, WBC differential) are usually in evidence. Aggressive antibiotic coverage with special focus on the Enterobacteriaceae is indicated along with dietary manipulation and biologics. Class 1B Naïve Crohn's Disease: Permanent tissue damage secondary of bacterial replication. Eradication of smoldering anaerobic infection allows for healing by fibrosis.

\section{Class 2 Crohn's Disease}

Class 2A crohn's disease: Ileocecal bowel penetration into the cul-de-sac with secondary cutaneous drainage. In this situation, two sites for concomitant therapeutic intervention are in play:
a. the ileocecal area and
b. the infected fistulous tract

Class 2B crohn's disease: Bowel-to-bowel anastomosis: Reestablishment of mucosal integrity is superseded by the need 
for aggressive anaerobic antibiotic coverage and institution of nutrition enhancement in anticipation of surgical removal of diseased tissue.

\section{Class 3 Crohn's Disease}

Class 3 crohn's disease: Prior surgical removal of diseased bowel. Class 3 designation identifies afflicted individuals who are at heightened risk of overwhelming sepsis and death. Prior surgery can reposition the bowel such that subsequent bowel penetration results in the spillage of fecal material into the peritoneal cavity rather than the cul-de-sac. Once diseased tissue has been removed and the small intestines repositioned, disruption of the immunemediated process is a clinical imperative.

\section{Demonstration of Relevance}

A prime example of where failure to match participants in therapeutic trials has resulted in therapeutic ambiguity is the evaluation of the efficacy of antituberculosis drugs for Crohn's disease. In individual cases, selective drug therapy had produced a rare permanent remission [5]. When clinical trials of antimicrobial compounds were collectively evaluated, the analysis identified an inconclusive, but positive, trend [6]. The role of antimicrobials for Crohn's disease failed to attain clinical reliance until Thomas Brody abandoned the quest for "evidence-based data" and concentrated on small, well-controlled clinical trials whose end titration point was sustained remission. By focusing therapy exclusively on Class 1A Crohn's disease, Brody has been able to document sustained remissions with documented mucosal healing [7].

His work has yet to garner the recognition due owing the welldocumented inability to recovery or identify with special stains MAP from diseased tissue containing MAP DNA. While these facts invalidated Crohn's disease as being an infectious disease due to MAP, the Brody data did was provide the confirmatory piece of the Hruska Postulate and its therapeutic extension. What has been postulated is that permanent remission requires destruction of the MAP templates which are constituted by MAP being in its spheroplastic form [8]. Why did some antimicrobial drugs work, and others did not reside with their respective mechanism-ofaction? To destroy MAP spheroplasts, the compound must disrupt RNA synthesis. Without proper alignment of study patient status, the role of selected antimicrobials would still be buried in data ambiguity.

\section{References}

1. Monif G R G (2015) The Hruska postulate of Crohn's disease. Med Hypothesis 85: 878-881.

2. Monif G R G (2018) The WHY? of Crohn's Disease. Adv Res Gastroenterol. Hepatol 10(5): 1-4.

3. Monif G R G (2017) An Infectious Disease Process within an ImmuneMediated Disease Process: Role of the Gastrointestinal Microbiota in Crohn's Disease. Adv Res Gastroenterol Hepatol 5: 1-2.

4. Monif G R G (2018) Understanding Therapeutic Concepts in Crohn's Disease. Clin Med Insights Gastroenterol 20: 1-3.

5. Warren J E, Rees H, Cox T (1986) Dietary Manipulation in Crohn's Disease? N Engl J Med 214: 182.

6. Selby W, Palvi P, Crotty B, Tim Florin, Graham Radford Smith, et al. (2007) Two-year combination antibiotic therapy with clarithromycin, rifabutin, and clofazimine for Crohn's disease. Gastroenterol 132: 2313-2319.

7. Agrawal G, Clancy A, Sharma R (2020) Targeted Combination Antibiotic Therapy Induces Remission in Treatment-Naïve Crohn's Disease: A Case Series. Microorganisms 8: 371-375.

8. Monif G R G (2020) MAP template controlling Crohn's disease? Med Hypothesis 138: 1-2. 